\title{
REAL-TIME LARGE SCALE 3D RECONSTRUCTION BY FUSING KINECT AND IMU DATA
}

\author{
J. Huai ${ }^{\mathrm{a}}$, Y. Zhang ${ }^{\mathrm{a}}$, A. Yilmaz ${ }^{\mathrm{a}, *}$ \\ ${ }^{a}$ Dept. of Civil, Environmental and Geodetic Engineering Dept, The Ohio State University \\ 2036 Neil Avenue, Columbus, OH 43210 USA - (huai.3, zhang.2669, yilmaz.15)@ osu.edu
}

Commission III, WG III/3

KEY WORDS: KinectFusion, IMU, SIFT Odometry, Large Scale Reconstruction

\begin{abstract}
:
Kinect-style RGB-D cameras have been used to build large scale dense 3D maps for indoor environments. These maps can serve many purposes such as robot navigation, and augmented reality. However, to generate dense 3D maps of large scale environments is still very challenging. In this paper, we present a mapping system for 3D reconstruction that fuses measurements from a Kinect and an inertial measurement unit (IMU) to estimate motion. Our major achievements include: (i) Large scale consistent 3D reconstruction is realized by volume shifting and loop closure; (ii) The coarse-to-fine iterative closest point (ICP) algorithm, the SIFT odometry, and IMU odometry are combined to robustly and precisely estimate pose. In particular, ICP runs routinely to track the Kinect motion. If ICP fails in planar areas, the SIFT odometry provides incremental motion estimate. If both ICP and the SIFT odometry fail, e.g., upon abrupt motion or inadequate features, the incremental motion is estimated by the IMU. Additionally, the IMU also observes the roll and pitch angles which can reduce long-term drift of the sensor assembly. In experiments on a consumer laptop, our system estimates motion at $8 \mathrm{~Hz}$ on average while integrating color images to the local map and saving volumes of meshes concurrently. Moreover, it is immune to tracking failures, and has smaller drift than the state-of-the-art systems in large scale reconstruction.
\end{abstract}

\section{INTRODUCTION}

In recent years visual simultaneous localization and mapping (SL$\mathrm{AM}$ ) has focused more on real-time solutions for dense mapping with hand-held cameras, which have broad applications in navigation, semantic mapping and robotics. To this end, multi-view stereo (MVS) [Furukawa and Ponce, 2010] achieved relatively dense reconstruction with accurate camera tracking. However, with cameras that does not sense depth, it is challenging to reconstruct surfaces without ambient light or sufficient texture. In contrast, RGB-D cameras like a Kinect, can capture color images along with the corresponding depth information at high rates. Following the advent of RGB-D sensors, a plethora of approaches have been proposed to solve for dense 3D mapping. A typical dense 3D mapping system consists of three major components: (1) Camera tracking based on the spatial alignment of consecutive frames; (2) Live scene reconstruction based on estimated camera pose and RGB-D data; (3) Loop closure detection and pose graph optimization.

To track the RGB-D sensor reliably, previous methods [Kerl et al., 2013]- [Endres et al., 2014] only relied on RGB-D data to estimate incremental motion. In environments with well distributed $2 \mathrm{D}$ and $3 \mathrm{D}$ features, visual odometry based solely on RGB-D data can be very accurate. However, it is prone to failures in scenes of few features and depth variations. In contrast, inertial sensors does not have such requirements on surroundings in localization. They have been used successfully in conjunction with visual sensors for terrestrial navigation [Kelly and Sukhatme, 2011]. An inertial measurement unit (IMU) can continuously estimate the motion with high frequency and small latency compared to visual sensors, but a consumer-grade IMU is subject to large drift over time. Such a drift can be constrained by the visual odometry estimates when the environment of rich features is traversed. Thus, it is highly desirable to fuse visual and inertial cues.

\footnotetext{
* Corresponding author
}

This paper combines the ICP algorithm [Besl and McKay, 1992], SIFT-based visual odometry [Lowe, 2004], and IMU-based tracking, to precisely and continuously estimate Kinect poses. This method does not assume good feature association and small motion, thus promises to improve continuity and quality of $3 \mathrm{D}$ reconstruction. To begin with, the relative orientation between the Kinect depth camera and the IMU was calibrated. During the algorithm's execution, the ICP is utilized at all epochs; the SIFT odometry is only performed when the ICP algorithm fails; when both break down, the IMU tracking module acts as a fall-back solution. As for the IMU module, an extended Kalman filter (EKF) propagates the IMU states with inertial readings, and corrects them by using inclinations observed by accelerometers, and position observations from ICP or SIFT odometry. Finally, detected loop closure provides further constraints to the map of which the pose graph is then optimized.

The following section discusses related work. Next, we introduce our 3D reconstruction system in Section 3. Then, experiments and results are presented in Section 4. Finally, Section 5 concludes this paper.

\section{RELATED WORK}

Many latest 3D reconstruction systems use RGB-D sensors like Kinect, to capture RGB images and depth data of the scene. One seminal system for real-time tracking and mapping is KinectFusion [Izadi et al., 2011], which proposes real-time dense surface mapping and tracking [Newcombe et al., 2011]. In the KinectFusion system, a surface model within a volume is generated and refined by recursively integrating depth frames. For each depth frame, its pose in the volume is determined by the coarse-to-fine ICP algorithm. Thanks to the surface model that serves as the reference, $3 \mathrm{D}$ reconstruction is achieved with robustness and great details. However, this system can only map small areas in real 
time due to high computational resource demand.

The Kinfu system [Rusu and Cousins, 2011] extends the KinectFusion to large scale reconstruction by using volume shifting and volume slice extraction. However, after going through several volumes, significant tracking drift may be observed especially at the loop closures. Moreover, the ICP algorithm for tracking often breaks down with rapid motion or in planar scenes, such as corridor.

To reduce drift and overcome such tracking failures, Kintinuous [Whelan et al., 2013] combines ICP and RGB-D tracking algorithms to estimate poses. Then they adopt deformation-based loop closure for large scale dense RGB-D SLAM [Whelan et al., n.d.]. In a similar spirit, RTAB-Map [Labbé and Michaud, 2011] used sparse features extracted from RGB images to aid ICP tracking. Moreover, it achieved large scale multi-session graph-based SLAM, which used online loop closure detection and graph-based map optimization. Shortly, [Henry et al., 2012] extracted sparse features from two consecutive color images and estimated an initial incremental motion with RANSAC-based alignment. The initial motion is then used to initialize the ICP estimation. With an off-line sparse bundle adjustment, highly accurate dense maps represented by surfels were achieved.

However, the tracking modules of these systems may fail in cases of few features. To estimate motion without failures, an inertial sensor is highly advantageous in combination with a Kinect. The literature on visual-inertial fusion teems with successful applications [Jones and Soatto, 2011]- [Kelly and Sukhatme, 2011]. As for fusing data collected by a Kinect and an IMU, [Nießner et al., 2014] proposed a method to robustly estimate inter-frame motion by incorporating inertial data. However, its effectiveness was only demonstrated on small areas.

Prior to combining visual and inertial cues, precise inter-sensor calibration is imperative. [Lobo and Dias, 2007]- [Mirzaei and Roumeliotis, 2008] provided basic approaches for calibrating offthe-shelf cameras and inertial sensors. The continuous approach proposed in [Furgale et al., 2013] with open source is a handy and accurate tool for IMU-camera calibration.

\section{EXTENDED KINECTFUSION FOR LARGE SCALE RECONSTRUCTION}

\subsection{Preliminaries}

Assume the RGB and depth cameras are calibrated well, then for most pixels in the RGB image, their depth can be retrieved from the depth frame captured by the depth camera. Thus, we can reconstruct a point $\mathbf{p}$ from its coordinates in the depth frame $\boldsymbol{u}=(u, v, 1)^{T}$, and the corresponding depth measurement $d(\boldsymbol{u})$ as follows:

$$
\boldsymbol{p}=\left[\begin{array}{c}
d(\boldsymbol{u}) K^{-1} \boldsymbol{u} \\
1
\end{array}\right]
$$

where $K$ is the intrinsic matrix of the depth camera. Note as points are computed with regard to depth camera frames, so are the incremental motions.

In order to project a world point, $\mathbf{p}_{W}$ onto the depth frame, it is first transferred from the world frame, $W$, to the current depth camera frame, $C$, through a rigid body transformation, $E_{C W}$, as in equation 2 .

$$
E_{C W}=\left[\begin{array}{cc}
R & t \\
\mathbf{0}^{T} & 1
\end{array}\right] \in S E(3) \quad \boldsymbol{p}_{C}=E_{C W} \boldsymbol{p}_{W}
$$

$E_{C W}$ also represents the camera pose with regard to the world frame. Then the point is projected onto the depth frame through a pinhole model as in equation 3 .

$$
\boldsymbol{u}=\operatorname{proj}\left(\begin{array}{c}
x \\
y \\
z \\
1
\end{array}\right)_{C}=\left(\begin{array}{l}
u_{0} \\
v_{0}
\end{array}\right)+\left[\begin{array}{cc}
f_{u} & 0 \\
0 & f_{v}
\end{array}\right]\left(\begin{array}{c}
\frac{x}{z} \\
\frac{y}{z}
\end{array}\right)
$$

Changes to the camera pose can be represented by a $4 \times 4$ camera motion $M$, which is also a member of $S E(3), E_{C^{\prime} W}=$ $M E_{C W}=\exp (\boldsymbol{\mu}) E_{C W}$. Here $\boldsymbol{\mu}$ is the minimal representation of $M$ in $S E(3), \boldsymbol{\mu}=\left[\begin{array}{ll}t & \omega\end{array}\right]^{T}$ where $\boldsymbol{\omega}$ represents the rotation part, and $t$ the translation part.

\subsection{Overview of Fusing the Kinect and IMU data}

This paper presents an extended KinectFusion system that fuses measurements from a Kinect and an IMU for tracking and mapping. The tracking component integrates three sources of odometry information, IMU, ICP, and SIFT odometry. The IMU module integrates IMU readings to predict Kinect attitudes, and corrects IMU biases and attitudes by using accelerometer readings and position observations from ICP and SIFT odometry. The rotation between two consecutive frames is predicted by the IMU module as follows. We timestamp frames and pose estimates from the IMU with the CPU clock time. These pose estimates are stored in a buffer, from which pose estimates closest to both frames in time can be retrieved and used to compute the inter-frame rotation. The inter-frame translation can also be computed, however, empirically a low cost MEMS IMU only estimates relative rotation reliably. With such a rotation estimate, the ICP algorithm tries to align the current depth frame and the predicted surface from the reconstructed surface model. Once ICP concludes, the incremental rotation by the IMU module can be used to check the validity of its motion estimate. If ICP fails, SIFT features are extracted and matched between these two frames. Thanks to the depth frames, these features form 3D point correspondences. Then, the SIFT odometry module tries to estimate motion by minimizing discrepancies between 3D point matches, again initialized and checked with the rotation estimate from the IMU. If SIFT odometry fails too, then the incremental motion is set as that of the IMU. Otherwise, the updated camera position is sent to the IMU module as observation to correct IMU states. However, empirically the IMU states do not benefit much from such observations. In addition, since the Kinect often moves with small acceleration, the IMU can roughly sense the gravity direction, thus constraining roll and pitch drift in the long run. As a result, the roll and pitch estimated by the IMU can be used to correct Kinect poses. Figure 1 shows the work flow and heuristics of the whole extended KinectFusion system.

\subsection{Large Scale KinectFusion}

In general, large scale reconstruction is achieved by volume shifting and concatenating multiple volumes. Both earlier methods Kinfu [Rusu and Cousins, 2011] and Kintinuous [Whelan et al., 2013] start a new volume once the distance between the previous volume center and current camera position exceeds a threshold. To this end, we use both the Euclidean distance and the number of pixels in the depth frame that are matched to the surface model. Therefore, both large displacement and large rotation will result in creating a new volume. For a new volume, its center sits at the optical center of the depth frame which is captured upon its creation. In our tests, volumes are generated, shifted, and saved online. During execution, loop closure constraints are computed using the combined odometry method discussed in Section 3.2 


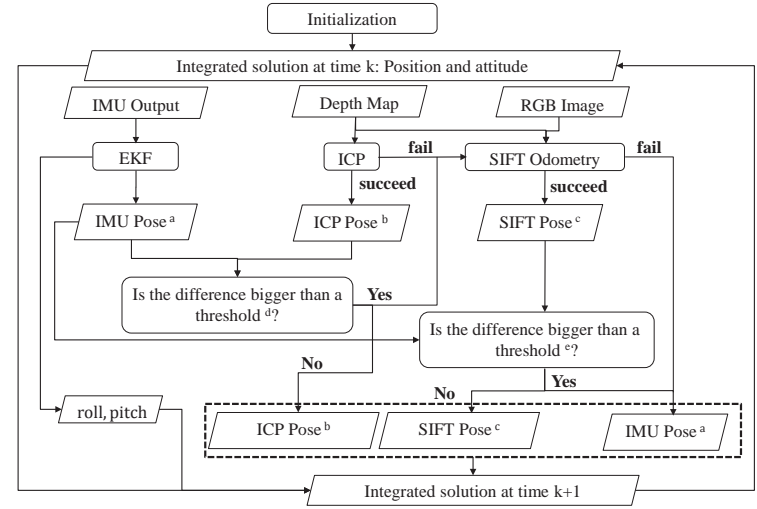

Figure 1: Workflow of extended KinectFusion system. (a) We only use the rotation of IMU from time $k$ to $k+1:\left\{\omega_{C_{k+1} C_{k}}^{I M U}, 0\right\}$; (b) The camera pose estimated by ICP contains the rotation and translation: $\left\{\omega_{C_{k+1} C_{k}}^{I C P}, t_{C_{k+1} C_{k}}^{I C P}\right\}$; (c) The camera pose estimated by SIFT odometry is $\left\{\omega_{C_{k+1} C_{k}}^{S I F T}, t_{C_{k+1} C_{k}}^{S I F T}\right\}$; (d) Check if the difference between incremental rotation estimated by ICP and that by the IMU module is greater than a threshold: $\| \omega_{C_{k+1} C_{k}}^{I C P}-$ $\omega_{C_{k+1} C_{k}}^{I M U} \|>T_{\omega}$; (e) Check if the difference between incremental rotation estimated by the SIFT odometry and that by the IMU module is greater than a threshold: $\left\|\omega_{C_{k+1} C_{k}}^{S I F T}-\omega_{C_{k+1} C_{k}}^{I M U}\right\|>$ $T_{\omega}$.

given detected revisits. With these constraints, the pose graph in terms of $S E(3)$ of the map is optimized with g2o [Kümmerle et al., 2011]. Finally, using these optimized poses, volumes of point clouds or meshes are updated and combined to make the large scale map.

\subsection{SIFT Odometry}

Similar to the IMU module, the SIFT odometry provides incremental motion between two depth frames, utilizing both depth and color frames. It is invoked only when ICP fails and the color and depth frames are well synchronized. The current color frame is firstly mapped to its synchronized depth frame, so that most pixels in the color frame correspond to some points in the depth coordinate system. Secondly, SIFT interest points are extracted from the current and previous color images and matched to obtain correspondences. For this step, we adopted SiftGPU [Wu, 2007]. Thirdly, for each correspondence, which is associated with a $3 \mathrm{D}$ point, its location in each color frame is mapped to a depth pixel in the respective depth frame through the calibration parameters. If both depth pixels have valid measurements, coordinates of the point observed in the previous $(C)$ and current $\left(C^{\prime}\right)$ depth coordinate system, $\boldsymbol{p}_{C}$ and $\boldsymbol{p}_{C^{\prime}}$, respectively, can be computed by equation 1 . Given a set of point matches (at least three), $S=\left\{\left(\boldsymbol{p}_{j C}, \boldsymbol{p}_{j C^{\prime}}\right), j=0, \ldots, n-1, n>2\right\}$, the relative camera motion $E_{C^{\prime} C}$ can be obtained by iteratively updating it by a small amount $\boldsymbol{\mu}$, i.e., left-multiplication with a $4 \times 4$ camera motion matrix, $\exp (\boldsymbol{\mu})$. The camera pose update, $\boldsymbol{\mu}$, can be computed with the weighted least squares by minimizing a robust objective function of discrepancies. To begin with, $E_{C^{\prime} C}$ is initialized as identity. Then in each iteration, the following calculations are performed:

1) For points in the previous frame, predict their positions in the current frame, compute the discrepancies, $e_{j}$, and their weights which are determined by $\operatorname{Obj}\left(e_{j}^{2}, \sigma_{T}^{2}\right)$, the Tukey bi-weight objective function, where $\sigma_{T}$ is a median-based estimate of the stan- dard deviation of these discrepancies:

$$
\begin{gathered}
e_{j}=\boldsymbol{p}_{j C^{\prime}}-E_{C^{\prime} C} \boldsymbol{p}_{j C} \\
\boldsymbol{\mu}=\underset{\boldsymbol{\mu}}{\operatorname{argmin}} \sum_{j \in S} \operatorname{Obj}\left(e_{j}^{2}, \sigma_{T}^{2}\right) e_{j}^{2}
\end{gathered}
$$

2) Optimize $\boldsymbol{\mu}$ by using the weighted least squares method. The Jacobian for each predicted point, $p=\left(E_{C^{\prime} C} \boldsymbol{p}_{j C}\right)_{1: 3}$ are computed as follows:

$$
J_{j}=\left.\frac{\partial \exp (\boldsymbol{\mu}) E_{C^{\prime} C} \boldsymbol{p}_{j C}}{\partial \boldsymbol{\mu}}\right|_{\boldsymbol{\mu}=0}=\left[\begin{array}{ll}
I_{3} & -p \times
\end{array}\right]
$$

Here $p \times$ denotes the skew matrix generated from the 3-vector $p$. Concatenating all $J_{j}, \operatorname{Obj}\left(e_{j}^{2}, \sigma_{T}^{2}\right)$, and $e_{j}$, we can get the whole Jacobian $J$, weight matrix $W$, and error vector e, respectively. The optimal $\boldsymbol{\mu}$ is the solution for the Gauss-Newton equation $J^{T} W J \mu=J^{T} W \mathbf{e}$.

3) Update $E_{C^{\prime} C}$ by applying $\boldsymbol{\mu}$, i.e., $\exp (\boldsymbol{\mu}) E_{C^{\prime} C}$.

\subsection{Calibration of the Kinect and IMU Rig}

In order to use the incremental motion estimate from the IMU module, the transformation between Kinect cameras and the IMU needs to be known. The calibration between the RGB camera and the depth camera is done in factory and relevant parameters are written in hardware. Therefore, we only have to calibrate for the relative transformation between the depth camera and the IMU. In our tests, we used a low cost MEMS IMU, Steval-MKI062V2 developed by STMicroelectronics. It is rigidly attached to the Kinect as shown in Figure 2. Since both the Kinect depth camera and IMU can measure rotation accurately, it is easy to estimate their relative rotation, $R_{C S}$.

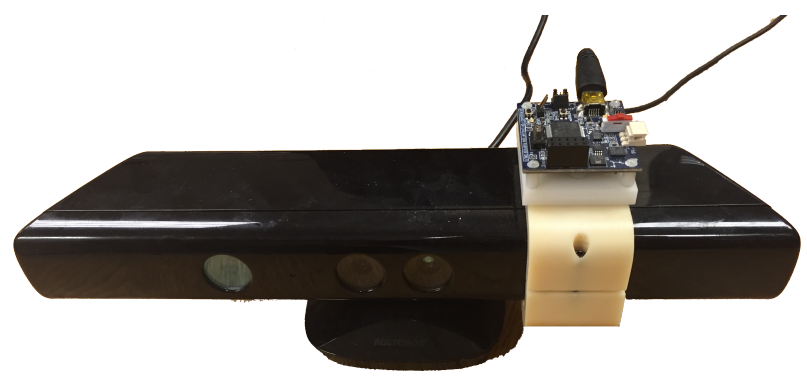

Figure 2: Combination of Kinect and IMU sensors.

The rotation calibration was conducted in a small workspace with rich 3D features, so that ICP can reliably estimate the attitude of the depth camera. First, synchronized data from the IMU and Kinect are collected. For a specific epoch, $k$, it includes the angular rate reading from gyros $\tilde{\omega}_{k}^{S}$ expressed in the IMU sensor frame, and the rotation matrix from the ICP algorithm, $R_{W C_{k}}$.

Considering that the calibration procedure takes only a few minutes, we assume that errors in $\tilde{\omega}_{k}^{S}$ consist of a constant bias and Gaussian noise. As a result, the constant bias can be estimated as the average of gyro readings in the static mode. Subtracting this bias from $\tilde{\omega}_{k}^{S}$ leads to estimated angular rates in the IMU frame, $\widehat{\omega}_{k}^{S}$.

Secondly, using the rotation matrices from ICP, the angular rate of the Kinect and IMU rig expressed in the depth camera frame, $\widehat{\omega}_{k}^{C}$, can be approximated by:

$$
[t(k+1)-t(k)] \widehat{\omega}_{k}^{C} \times=R_{W C_{k+1}}{ }^{T} R_{W C_{k}}-I_{3} .
$$


where $\widehat{\omega}_{k}^{C}=R_{C S} \widehat{\omega}_{k}^{S}$ for all $k$, and the best fit of $R_{C S}$ can be estimated from the SVD of $H=\sum_{k=1}^{n} \widehat{\omega}_{k}^{S}\left(\widehat{\omega}_{k}^{C}\right)^{T}$. The least squares solution is found as $R_{C S}=U V^{T}$ given $H=U \Sigma V^{T}$ [Arun et al., 1987].

The estimation of the lever arm between the Kinect cameras and the IMU is rather involved. The coarse value of lever arm between the RGB camera and the IMU is manually measured. Then several calibration tests were conducted with the Kalibr package [Furgale et al., 2013]. Due to the rolling shutter of the RGB camera, the lever arm estimate in different tests fluctuated about the manually measured values. As a result, the mean of these estimates was used as the lever arm in later experiments.

\subsection{IMU Odometry Based on EKF}

The IMU tracking module maintains states consisting of position and velocity of the IMU in the world frame, $\mathbf{p}_{s}^{W}$ and $\mathbf{v}_{s}^{W}$, respectively, rotation from the world frame to the IMU frame expressed by a quaternion, $q_{S W}$, accelerometer biases $\mathbf{b}_{a}$ and gyro biases $\mathbf{b}_{g}$. In sum, the whole state vector is

$$
\mathbf{x}=\left\{\mathbf{p}_{s}^{W}, \mathbf{v}_{s}^{W}, q_{S W}, \boldsymbol{b}_{a}, \boldsymbol{b}_{g}\right\}
$$

The world frame's origin is anchored at the origin of the IMU frame at the start of an experiment. Its $Z$ axis is aligned with the local gravity vector. Its other two axes are defined such that the rotation between the IMU frame at the beginning of an experiment and the world frame involves zero yaw angle. The realization of this world frame is achieved by using zero velocity update (ZUPT) and accelerometer readings at a test's start. The propagation model for the IMU states are formulated in equation 7.

$$
\begin{aligned}
\frac{d \mathbf{p}_{s}^{W}}{d t} & =\mathbf{v}_{s}^{W} \\
\frac{d \mathbf{v}_{s}^{W}}{d t} & =R_{W S} \mathbf{a}^{S}+\mathbf{g}^{W}-2 \omega_{i e}^{W} \times \mathbf{v}_{s}^{W} \\
\dot{R}_{S W} & =\left(\omega_{i e}^{S}-\omega_{i s}^{S}\right) \times R_{S W}
\end{aligned}
$$

where $\omega_{i e}^{S}$ represent the Earth rotation rate with respect to the inertial frame expressed in the IMU sensor frame, and $\mathbf{g}^{W}=$ $[0,0, g]^{T}$ is the nominal local gravity. Assume constant gravity in the local area, ignore the Earth rotation, and model accelerometer and gyro biases as random walk processes, the linearized continuous transition model is derived and given by:

$$
\begin{aligned}
\frac{d \delta \mathbf{p}_{s}^{W}}{d t} & =\delta \mathbf{v}_{s}^{W} \\
\frac{d \delta \mathbf{v}_{s}^{W}}{d t} & =-R_{W S}\left(\tilde{\mathbf{a}}^{S}-\mathbf{b}_{a}\right) \times \psi^{S}+R_{W S}\left(\mathbf{b}_{a}+\mathbf{n}_{a}\right) \\
\dot{\psi} S & =-\left(\tilde{\omega}_{i s}^{S}-\mathbf{b}_{g}\right) \times \psi^{S}+\mathbf{b}_{g}+\mathbf{n}_{g} \\
\tilde{R}_{S W} & =\left(1-\psi^{S} \times\right) R_{S W} \quad \dot{\mathbf{b}}_{a}=\mathbf{n}_{a w} \quad \dot{\mathbf{b}}_{g}=\mathbf{n}_{g w}
\end{aligned}
$$

We use EKF to correct the states given observations such as ZUPT (equation 9) and measured gravity direction by accelerometers (equation 10). Because the small acceleration assumption does not always hold true, we apply the gravity observation model to constrain roll and pitch only when the magnitude of the estimated acceleration, $\left\|\tilde{\mathbf{a}}^{S}-\mathbf{b}_{a}\right\|$, deviates from $g$ less than a cutoff value (our tests used $1.5 \mathrm{~m} / \mathrm{s}^{2}$ ):

$$
\begin{gathered}
\mathbf{0}=\boldsymbol{v}_{s}^{W}+\boldsymbol{n}_{v} \\
\mathbf{g}^{W}=-R_{W S}\left(\tilde{\mathbf{a}}^{S}-\mathbf{b}_{a}\right)+\mathbf{n}_{g}
\end{gathered}
$$

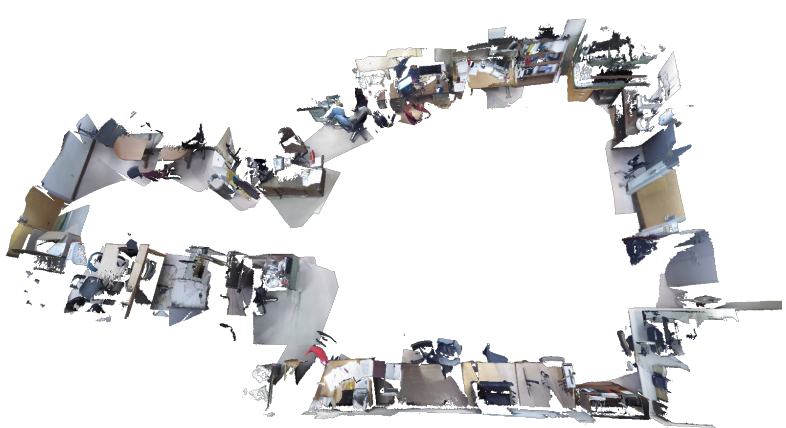

(a)

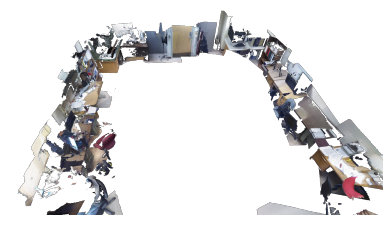

(b)

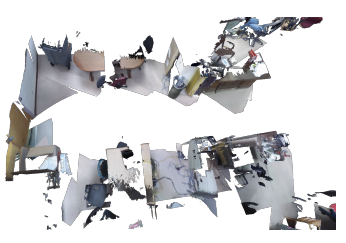

(c)
Figure 3: Meshes for the office after loop closure. The upper picture shows the whole loop of the office and the lower two views represent the details of the two small loops. Since we didn't capture sufficient data in the second small loop, there are some missing parts here.

\section{EXPERIMENTS AND RESULTS}

Several indoor experiments are conducted to validate our method's advantages over other motion estimation methods, including areas with insufficient depth and color features. The proposed method runs in real time on a computer with a $2.4 \mathrm{GHz} \mathrm{CPU}$ and $8 \mathrm{~GB}$ RAM. Three experiments are presented: One was conducted in an large office of travel distance around $36 \mathrm{~m}$, one in a corridor of around $65 \mathrm{~m}$, and the last on several flights of stairs of around $83 \mathrm{~m}$. Each test was started with a static session of about 45 seconds to calibrate the IMU and to ensure the initial roll and pitch converged. In order to observe the drift effect, each test contained a loop. For comparison, state-of-the-art tracking methods, ICP and SIFT, were used to estimate motion for the datasets collected in three experiments. The ICP based approach broke down in areas without enough depth features, such as corridor and stairs. SIFT odometry was more robust to failures than ICP but often got rather coarse orientations in featureless areas. In contrast, our combined odometry (ICP+SIFT+IMU) ran through entire experiments smoothly and resulted in less drift for large scale reconstruction. We present the trajectories generated by the SIFT odometry, our combined odometry (ICP+SIFT+IMU) and the combined odometry with loop closure. The trajectory from ICP is not presented because it frequently failed due to insufficient depth features.

For the test in an office, the meshes of the office area after loop closure are shown in figure 3 . The two closer views at its second row illustrates details of meshes. The missing and discontinuous texture are primarily due to the volume shifting step as each volume is confined by a cube and cannot integrate texture beyond it. In the future, we will use a volume slicing and rotating technique as in Kintinuous [Whelan et al., 2013] to solve this problem. Figure 4 shows the view of trajectories in $\mathrm{X}-\mathrm{Z}$ plane of the depth camera frame at the test start.

Similarly, for the corridor test, figure 5 shows the meshes of corridor after loop closure. Again, its bottom row displays two closer view of the reconstructed scene. The $2 \mathrm{D}$ trajectories in the $\mathrm{X}-\mathrm{Z}$ 


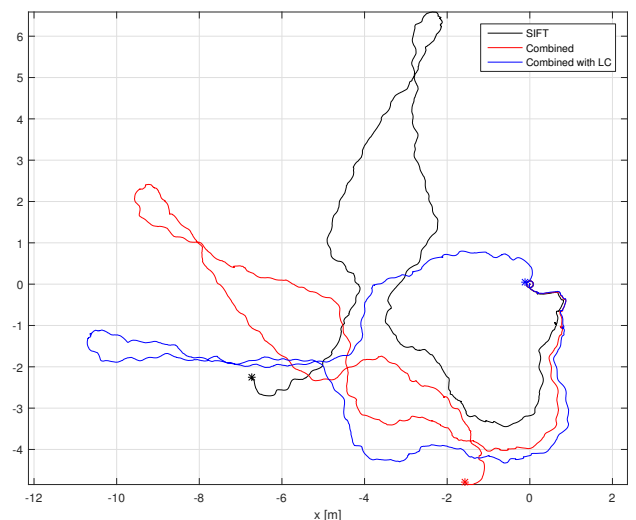

Figure 4: Trajectories projected to $\mathrm{X}-\mathrm{Z}$ plane of the depth camera frame at the start of the office test. "०" marks the starting point and "*" the finish point. Large orientation erros are observed in the SIFT odometry. Combined odometry gives better results, but still suffers in heading. This may be caused by many movements close to pure rotations in the test. The loop closure handles the orientation inconsistency very well.

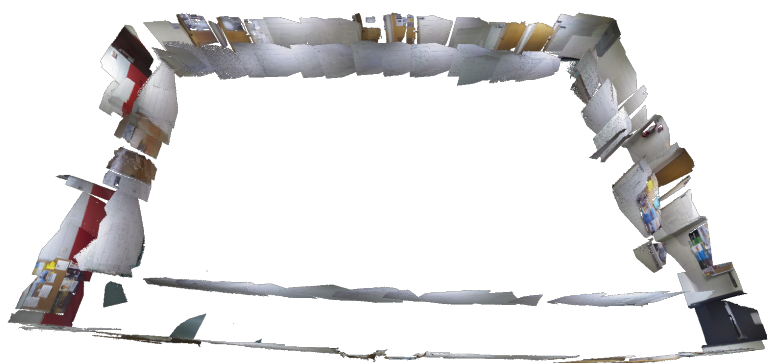

(a)

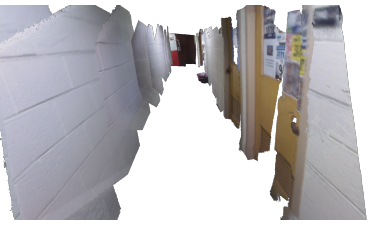

(b)

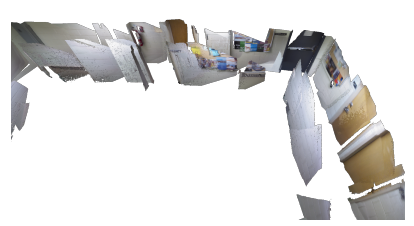

(c)
Figure 5: Meshes for the corridor after loop closure. The upper picture shows the whole loop of the corridor and the lower two views represent the inner view and one part of the loop. The missing and discontinuous texture are due to the volume shifting step as each volume is confined by a cube and cannot integrate texture beyond it.

plane are drawn in figure 6 . For the test in a stairway, the meshes of four flights after loop closures are depicted in figure 7. Figure 8 shows the corresponding trajectories.

\section{CONCLUSION}

We presented an extended KinectFusion system for real-time large scale 3D reconstruction by fusing Kinect and IMU data. In order to estimate sensor pose robustly, the ICP algorithm, SIFT odometry, and the IMU odometry are combined into a single pipeline. This combined odometry bridges tracking failures often experienced by methods only using RGB and depth information upon

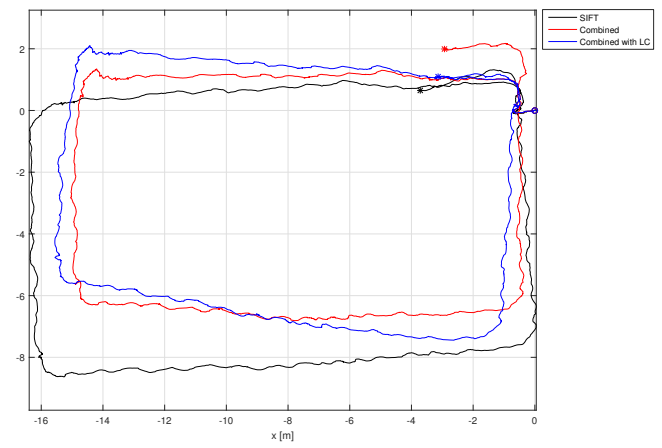

Figure 6: Trajectories projected to X-Z plane of the depth camera frame at the start of the corridor test. "o" marks the starting point and "*" the finish point. The SIFT odometry keeps the shape well despite a scale change. Combined odometry gives better results in orientation and scale. The loop closure removes the trajectory inconsistency.

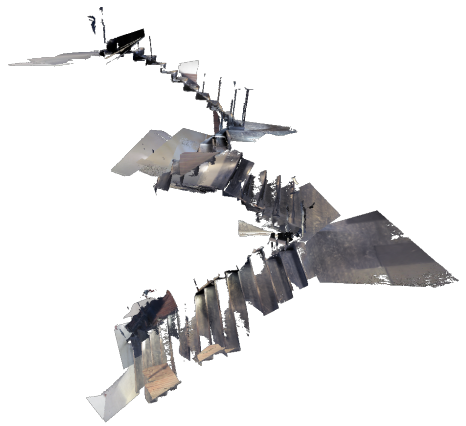

(a)

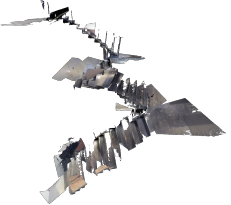

(b)

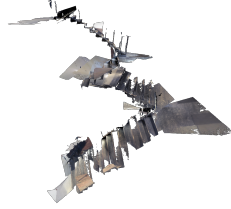

(c)
Figure 7: Meshes for stairs after loop closure. The upper picture shows the panorama of four flights of stairs and the lower two views represent the stairs to the second and fourth floor. The missing and discontinuous texture is partially caused by quick motion and hence inadequate color images.

abrupt camera motion or inadequate features. It is verified that the system we developed outperforms state-of-the-art methods in reducing drift and ensuring continuous motion estimates. Based on motion estimation from the combined odometry and loop closure, large scale reconstruction of good quality is achieved.

\section{REFERENCES}

Arun, K. S., Huang, T. S. and Blostein, S. D., 1987. Least-squares fitting of two 3-d point sets. Pattern Analysis and Machine Intelligence, IEEE Transactions on (5), pp. 698-700.

Besl, P. J. and McKay, N. D., 1992. A method for registration of 3-d shapes. IEEE transactions on pattern analysis and machine intelligence 14(2), pp. 239-256.

Endres, F., Hess, J., Sturm, J., Cremers, D. and Burgard, W., 


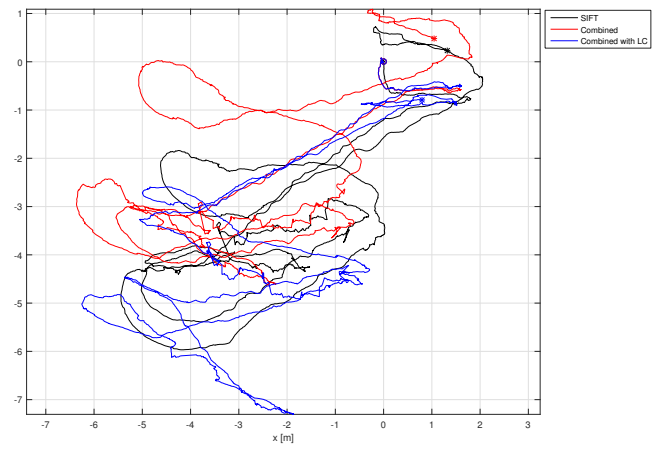

Figure 8: Trajectories projected to X-Y plane of the depth camera frame at the start of the stairway test. "o" marks the starting point and "*" the finish point. Combined odometry gives better results in orientation and scale. The loop closure removes the trajectory inconsistency.

2014. 3-d mapping with an rgb-d camera. Robotics, IEEE Transactions on 30(1), pp. 177-187.

Furgale, P., Rehder, J. and Siegwart, R., 2013. Unified temporal and spatial calibration for multi-sensor systems. In: Intelligent Robots and Systems (IROS), 2013 IEEE/RSJ International Conference on, IEEE, Tokyo, Japan, pp. 1280-1286.

Furukawa, Y. and Ponce, J., 2010. Accurate, dense, and robust multiview stereopsis. Pattern Analysis and Machine Intelligence, IEEE Transactions on 32(8), pp. 1362-1376.

Henry, P., Krainin, M., Herbst, E., Ren, X. and Fox, D., 2012. Rgb-d mapping: Using kinect-style depth cameras for dense $3 \mathrm{~d}$ modeling of indoor environments. The International Journal of Robotics Research 31(5), pp. 647-663.

Izadi, S., Kim, D., Hilliges, O., Molyneaux, D., Newcombe, R., Kohli, P., Shotton, J., Hodges, S., Freeman, D., Davison, A. et al., 2011. Kinectfusion: real-time $3 \mathrm{~d}$ reconstruction and interaction using a moving depth camera. In: Proceedings of the 24th annual ACM symposium on User interface software and technology, UIST '11, ACM, New York, NY, USA, pp. 559-568.

Jones, E. S. and Soatto, S., 2011. Visual-inertial navigation, mapping and localization: A scalable real-time causal approach. The International Journal of Robotics Research 30(4), pp. 407-430.

Kelly, J. and Sukhatme, G. S., 2011. Visual-inertial sensor fusion: Localization, mapping and sensor-to-sensor self-calibration. The International Journal of Robotics Research 30(1), pp. 56-79.

Kerl, C., Sturm, J. and Cremers, D., 2013. Dense visual slam for rgb-d cameras. In: Intelligent Robots and Systems (IROS), 2013 IEEE/RSJ International Conference on, IEEE, Tokyo, Japan, pp. 2100-2106.

Kümmerle, R., Grisetti, G., Strasdat, H., Konolige, K. and Burgard, W., 2011. g 2 o: A general framework for graph optimization. In: Robotics and Automation (ICRA), 2011 IEEE International Conference on, IEEE, Shanghai, China, pp. 3607-3613.

Labbé, M. and Michaud, F., 2011. Memory management for real-time appearance-based loop closure detection. In: Intelligent Robots and Systems (IROS), 2011 IEEE/RSJ International Conference on, IEEE, San Francisco, CA, USA, pp. 1271-1276.

Lobo, J. and Dias, J., 2007. Relative pose calibration between visual and inertial sensors. The International Journal of Robotics Research 26(6), pp. 561-575.
Lowe, D. G., 2004. Distinctive image features from scaleinvariant keypoints. International Journal of Computer Vision 60(2), pp. 91-110.

Mirzaei, F. M. and Roumeliotis, S., 2008. A kalman filter-based algorithm for imu-camera calibration: Observability analysis and performance evaluation. Robotics, IEEE Transactions on 24(5), pp. 1143-1156.

Newcombe, R. A., Izadi, S., Hilliges, O., Molyneaux, D., Kim, D., Davison, A. J., Kohi, P., Shotton, J., Hodges, S. and Fitzgibbon, A., 2011. Kinectfusion: Real-time dense surface mapping and tracking. In: Mixed and augmented reality (ISMAR), 2011 10th IEEE international symposium on, IEEE, Basel, Switzerland, pp. 127-136.

Nießner, M., Dai, A. and Fisher, M., 2014. Combining inertial navigation and icp for real-time $3 \mathrm{~d}$ surface reconstruction. Eurographics 2014-Short Papers pp. 13-16.

Rusu, R. B. and Cousins, S., 2011. 3d is here: Point cloud library (pcl). In: Robotics and Automation (ICRA), 2011 IEEE International Conference on, IEEE, Shanghai, China, pp. 1-4.

Whelan, T., Johannsson, H., Kaess, M., Leonard, J. J. and McDonald, J., 2013. Robust real-time visual odometry for dense rgb-d mapping. In: Robotics and Automation (ICRA), 2013 IEEE International Conference on, IEEE, Karlsruhe, Germany, pp. 5724-5731.

Whelan, T., Kaess, M., Leonard, J. J. and McDonald, J., n.d. Deformation-based loop closure for large scale dense rgb-d slam. In: Intelligent Robots and Systems (IROS), 2013 IEEE/RSJ International Conference on, Tokyo, Japan, pp. 548-555.

Wu, C., 2007. SiftGPU: A GPU implementation of scale invariant feature transform (SIFT). http://cs.unc.edu/ ccwu/ siftgpu. 